\title{
SCANNING OF OPTICAL FIELDS USING NEAR-FIELD SCANNING OPTICAL MICROSCOPY
}

The Near-field scanning optical microscopy is a high-resolution diagnostics for the optical field characterization. In our experiments we focus on the optical field characterization of semiconductor laser diodes based on multiple quantum well structure as well photonic crystal fibers. The parameters of optical field of laser diode in the near-and far-field zone as well the local spectral analysis of the quantum well laser devices in the near-field will be studied. The mode distribution across photonic crystal fiber was scanned using near-field scanning optical microscopy. This experimental technique in combination with an analysis of optical field in the near-field region could be an effective optical tool for the final diagnostics of laser devices and photonic crystal fibers.

\section{Introduction}

Near-field scanning optical microscopy (NSOM) is a promising diagnostics of optical microscopies, which can provide optical resolution better than the Rayleigh diffraction limit [1]. NSOM uses optical fiber tip with an aperture that is much smaller than the wavelength ( of the detected light. Typically, an optical fiber is shaped to a small tip with an aperture in the range of $100 \mathrm{~nm}$. The application of near-field imaging and spectroscopy to optical and optoelectronic devices provides subwavelength information on a device structure, performance and output properties [2, 3, 4]. The emission profile is obtained by coupling the emitted radiation into the fiber-tip moving along the investigated emitter. The nearfield properties could be determined by placing the fiber tip very close to the sample surface (in order of 10-100 nm).

Laser devices based on multiple quantum well (MQW) structures became attractive in last decade because of their excellent optical properties. We present the characterization of a MQW laser structure based on GaAs material with InAs monolayers. The mode spectrum of laser devices based on this structure as well band calculations were published in [5]. In this paper we focus on the characterization of InAs/GaAs MQW laser device using NSOM. The profile of optical field as well local spectral analysis of MQW laser diodes is directly investigated.

Photonic crystal fibers (PCF) are at the top of interest because of their distinct guiding mechanism of light. PCF consists of an array of microscopic air holes in the glass fiber reminiscent of a crystal lattice [6]. We employ NSOM diagnostics to characterize the lateral modes in the PCF.

\section{Experimental}

In the experimental part we focused on the preparation of high-quality optical fiber tips for NSOM investigations. The technology of etching in hydrogenfluorid acid and pulling in small flame, respectively, were used for the preparation of the high quality tapered optical fiber tips. A quality of fiber tips was examined by the Scanning electron microscopy (SEM). The near-field of the MQW laser structures and the mode shape of the fundamental and the higher-order lateral mode of PCF were investigated using the fabricated fiber tips.

The MQW laser structures have been grown by low-pressure metal-organic vapor phase epitaxy (MOVPE) on (001) oriented ntype GaAs substrates. The layer structure with the detailed waveguide arrangement is shown in fig. 1. The structure consists of a $150 \mathrm{~nm}$ n-doped GaAs buffer layer followed by $625 \mathrm{~nm}$ n-doped $\mathrm{Al}_{\mathrm{x}} \mathrm{Ga}_{1-\mathrm{x}} \mathrm{As}$ confinement layer, the MQW active region and $625 \mathrm{~nm}$ p-doped $\mathrm{Al}_{\mathrm{x}} \mathrm{Ga}_{1-\mathrm{x}} \mathrm{As}$. The structure was covered with a $15 \mathrm{~nm}$ GaAs layer. The $90 \mathrm{~nm}$ thick active region contains 9 InAs monolayers spatially separated by $2.5 \mathrm{~nm}$ thick GaAs barriers [5]. Al composition in $\mathrm{Al}_{\mathrm{x}} \mathrm{Ga}_{1-\mathrm{x}} \mathrm{As}$ confinement layers was varied to form the graded-index waveguide.

The gain guided metal stripe lasers were fabricated for the experimental emission studies. The stripes width of $5 \mu \mathrm{m}$ in $\mathrm{SiO}_{2}$ mask and $\mathrm{AuBe} / \mathrm{Ti} / \mathrm{Au}$ p-contact layer was deposited. The device fabrication was completed by thinning of the wafer to $150 \mu \mathrm{m}$ and evaporation of bottom $\mathrm{AuGe} / \mathrm{Au}$ n-contact. The samples were cleaved perpendicular to metal stripes to have cavity facets.

\footnotetext{
* D. Pudišr ${ }^{1}$, I. Martinček ${ }^{1}$, I. Turek ${ }^{1}$, M. Michalka ${ }^{2}$, J. Kováč, jr. ${ }^{3}$, V. Gottschalch ${ }^{4}$, D. Káčik ${ }^{1}$

${ }^{1}$ Dept. of Physics, Faculty of Electrical Engineering, University of Žilina, Velký diel, 010 26, Žilina, Slovakia,

${ }^{2}$ International Laser Centre, Ilkovičova 3, Bratislava 812 19, Slovakia,

${ }^{3}$ Dept. of Microelectronics, Faculty of Electrical Engineering and Information Technology, Slovak University of Technology, Ilkovičova 3, Bratislava, Slovakia

${ }^{4}$ Faculty of Chemical and Physics, University of Leipzig, Linnéstrasse 3-5, 04103 Leipzig, Germany, E-mail: pudis@fyzika.utc.sk
} 
For the NSOM investigations the laser diode was mounted on the micro-mechanical positioning stage. The beam profile was scanned in the vertical (in the growth direction) and lateral (in the plane of layer structure) direction in the front of the laser facet using the prepared optical fiber tips (Fig. 2). The resonator parameters of the laser diode are stripe length $d=235 \mu \mathrm{m}$, stripe width $w=22 \mu \mathrm{m}$.

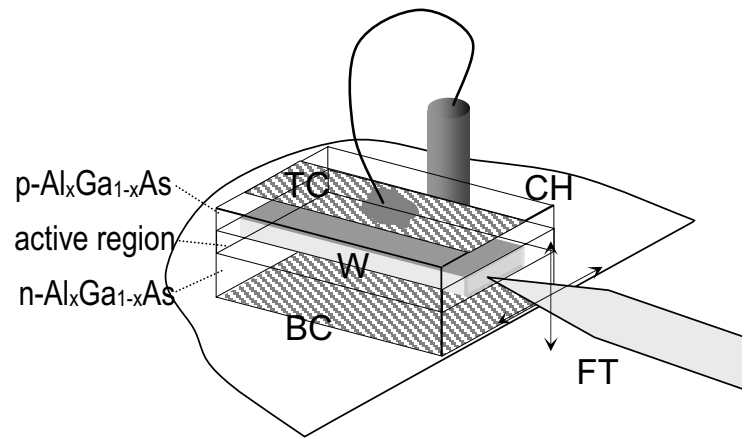

Fig.1 The laser diode and a detail of the NSOM experiment on the front laser facet; $\mathrm{CH}$, laser diode chip; FT, fibre-tip; TC, top contact;

$B C$, bottom contact; $W$, gain guided waveguide.

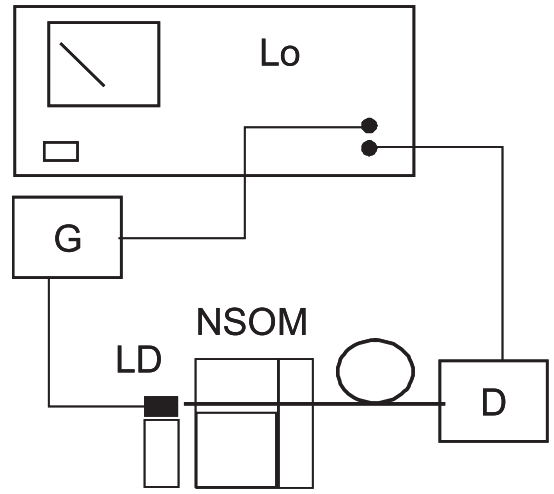

Fig. 2 Experimental setup of the NSOM experiment; LD, laser diode; NSOM, positioning stage; G, pulse generator; D, Si-detector; Lo, Lock-in amplifier. For the local spectral analysis the Si-detector and Lock-in amplifier by spectral analyzer was replaced.

The mode spectrum as well the near-field shape of stripe laser diode was investigated using the NSOM diagnostics and the spectral analyzer with the spectral resolution of $0.07 \mathrm{~nm}$. The operation spectral region of these quantum well laser diodes is in the

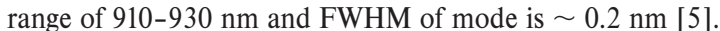

The optical near-field region of laser diode was recorded using the optical fiber tip mounted on the 3D mechanical positioning stage with step $50 \mathrm{~nm}$. The tip diameter used for field detection was better than $200 \mathrm{~nm}$ [7]. The fiber tip was placed close to the front laser facet $(<50 \mathrm{~nm})$. Two experimental setups were used to analyze the near-field shape and the laser emission spectra of the laser device: i) For the near-field shape investigations of the laser diode the experimental arrangement with Si-detector was used (Fig. 2).

ii) The spectral analysis measurements were investigated using the spectral analyzer Anritsu HS 9710B with spectral range of 0.6-1.7 $\mu$ m (Fig. 1) $[8,9]$.

The spectrum was analyzed using NSOM experiment at different positions of the fiber tip on the front laser facet. The detail scheme of an experiment is shown in Fig. 1. The mode laser spectrum was recorded at different vertical positions of the NSOM fiber tip on the front laser facet. The vertical step of the experiment was set to $300 \mathrm{~nm}$. The horizontal position of the fiber tip was set to get a maximal intensity.

Modes in PCF were excited using the InAs/GaAs MQW laser diode at wavelength $\lambda=923 \mathrm{~nm}$. In the experimental setup for mode investigation the PCF was fixed on the steady holder instead of the laser diode (Fig. 1). The optical field of two different lengths $150 \mathrm{~cm}$ and $7 \mathrm{~cm}$ of PCF was scanned to show the propagation of fundamental and higher-order lateral modes. The PCF under studies has the core diameter $\sim 13 \mu \mathrm{m}$, the average hole diameter $2.6 \mu \mathrm{m}$ and pitch $7.1 \mu \mathrm{m}[10]$.

\section{Experimental results}

Prepared optical fiber tips were studied by SEM Their quality was examined by scanning of optical field of MQW laser structures and PCF in the near-field region using NSOM arrangement.

From SEM images the fiber tip diameter was determined to be less than $100 \mathrm{~nm}$ for tapered fibers prepared using technology of pulling in small flame and less than $800 \mathrm{~nm}$ using etching technology. Detection properties of optical fibers prepared using both of technologies were compared from the measurements of the near-field profile of MQW laser diode. In the near-field profile scanned in the vertical direction the ghost peaks were observed for the etched fiber tips as is shown in fig. 4 . Their origin is probably caused by the surface inhomogenities arisen during the etching process. Therefore, tapered optical fibers prepared by pulling technology in the small flame were used in the forthcoming nearfield investigations.

The near-field investigation of the laser diode shows Gaussian shape of the optical field scanned in the vertical and horizontal direction (Fig. 5a). The light propagation in the $z$-axis is shown in Fig. $5 \mathrm{~b}$ as an intensity image and can be theoretically described by equation [4]

$$
W^{2}(z)=W_{0}^{2}\left[1+\left(\frac{z}{z_{0}}\right)^{2}\right],
$$

where $W_{0}$ is beam waist in the near-field region and $z_{0}$ is confocal parameter given by

$$
\mathrm{z} 0=\pi W_{0}^{2} / \lambda .
$$




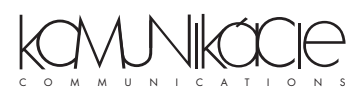

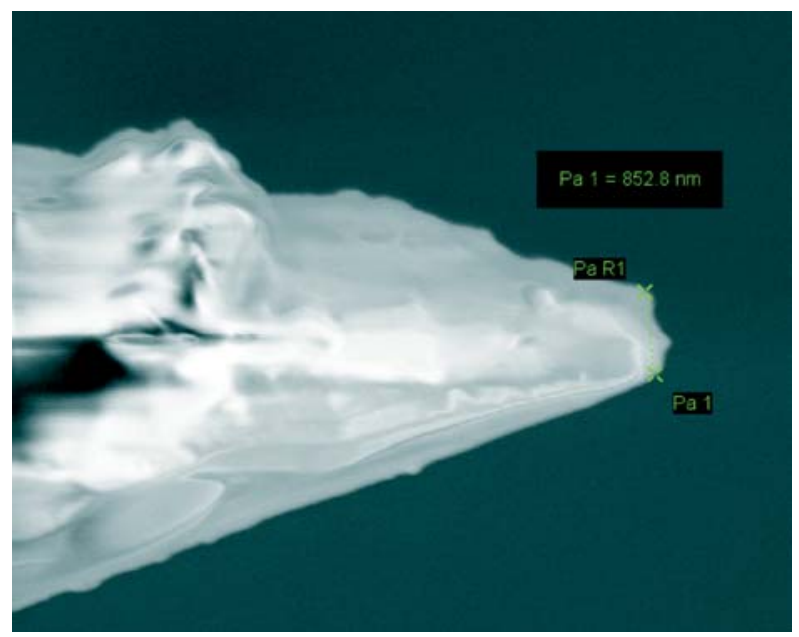

a)

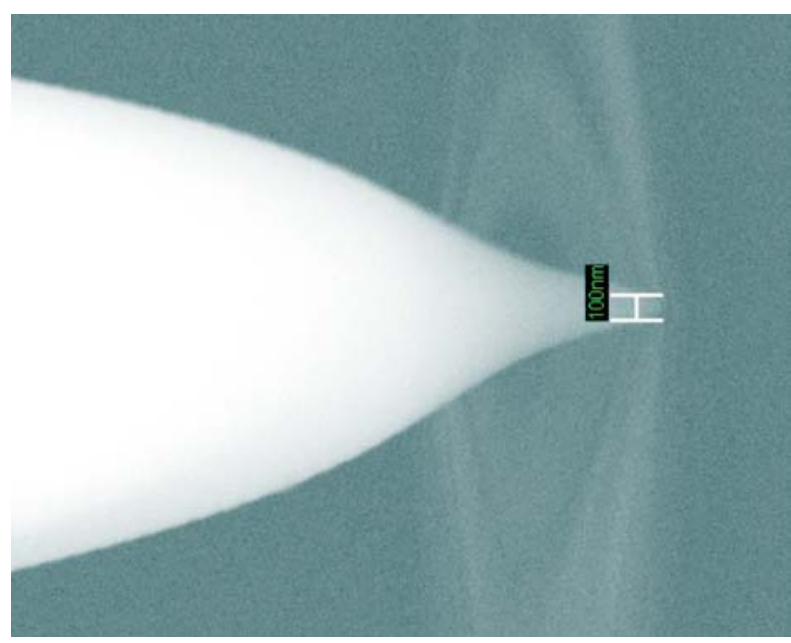

b)

Fig.3 The SEM image of the prepared fiber tips using a) etching and b) pulling in small flame.

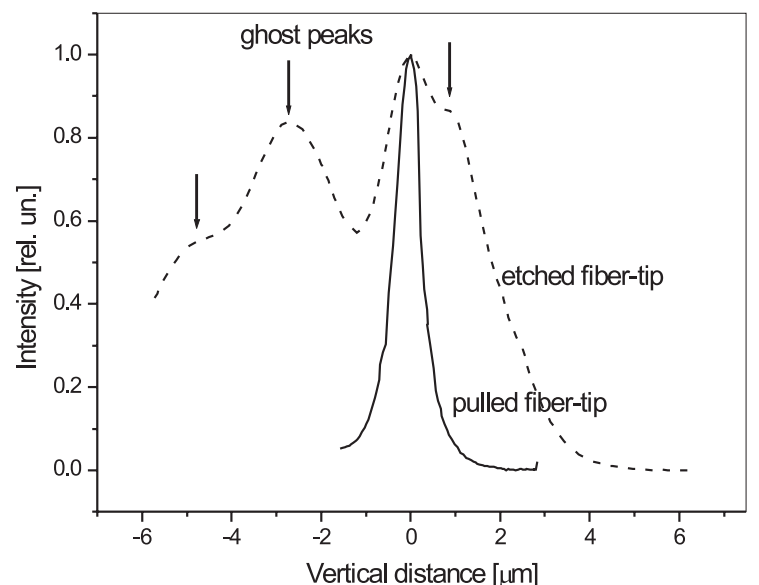

Fig. 4 Profile of the optical field of the laser structure recorded in the near-field region using the etched and pulled optical fiber tips.

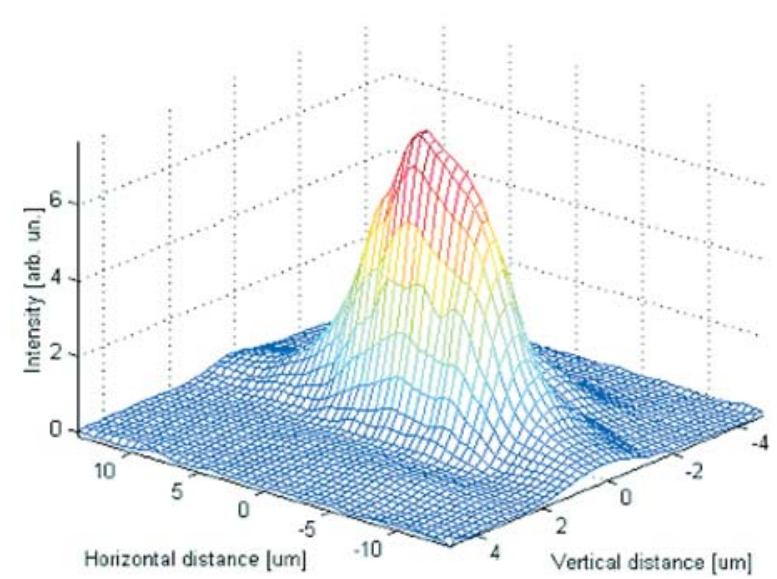

a)
The emission wavelength ( was determined from the spectral measurements to be $923 \mathrm{~nm}$. The divergence angle $\theta_{0}$ of the propagating light is a function of the emission wavelength and beam waist

$$
\theta_{0}=\lambda / \pi W_{0}
$$

From the measured optical field distribution in the near- and far-field region (Fig. 5a and 5b) the following parameters were estimated $2 W_{0}=1.97 \mu \mathrm{m}, z_{0}=3.30 \mu \mathrm{m}$ and $\theta_{0}=0.298 \mathrm{rad}$.

The measured mode spectrum of the laser diode is shown in Fig. 6a for different vertical positions of the fiber tip on the front laser facet. The vertical step of record was $300 \mathrm{~nm}$. For these vertical measurements the horizontal position of the fiber tip was set to obtain the maximum intensity of a signal. The corresponding density image of the mode spectrum distribution is shown in Fig.

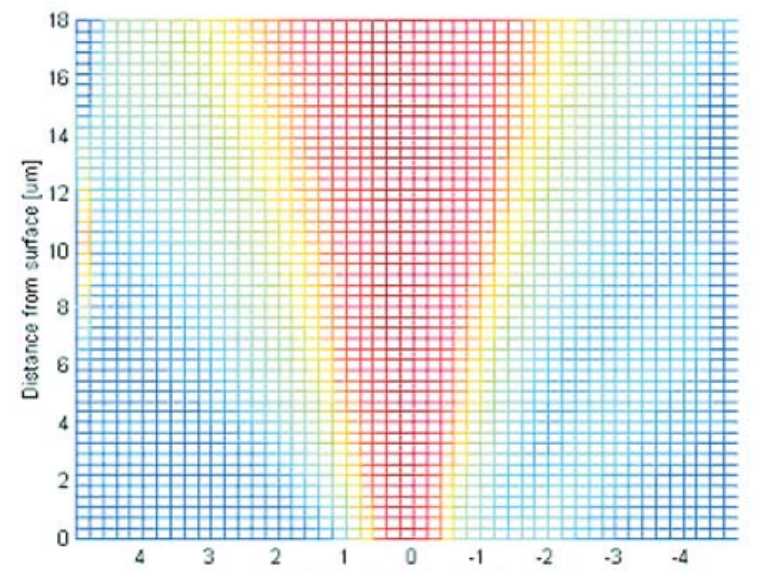

b)

Fig. 5a) The optical field scanned in the vertical and horizontal direction. b) Intensity image of the optical field distribution scanned in the vertical direction in different distances from the front laser facet. 
6b. Modes in the laser spectrum correspond to longitudinal modes for the Fabry-Pérot resonator. Their wavelength separation could be determined from the simple approach

$$
\Delta \lambda \approx \frac{\lambda^{2}}{2 n d},
$$

where $\lambda$ is the wavelength one of adjacent modes, $n$ is refractive index in the resonator and $d$ is the resonator length. The mode separation of the investigated laser diode for adjacent modes (maximum at wavelength $\lambda=919.8 \mathrm{~nm}$ ) was determined from measured spectra $\Delta \lambda=0.5 \mathrm{~nm}$. This experimental result corresponds to the theoretical calculation $\Delta \lambda=0.507 \mathrm{~nm}$, following equation (4) and taking into account the waveguide parameters of the laser diode, stripe length $d=235 \mu \mathrm{m}$ and refractive index of active region $n_{(\text {GaAs })}=3.55$

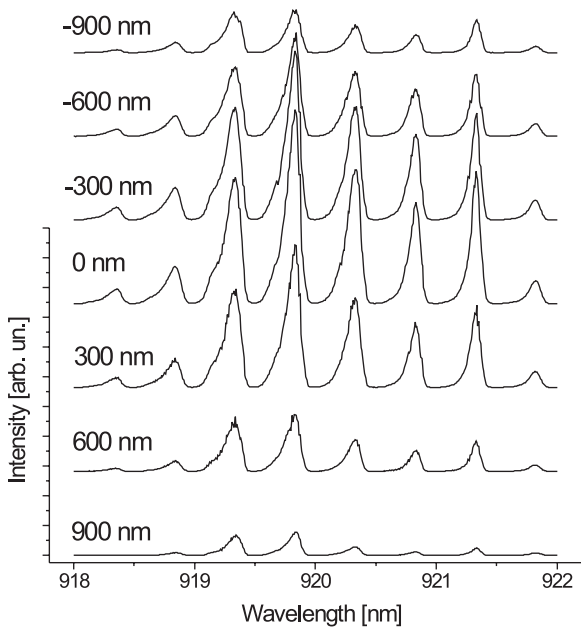

a)
In all range of the vertical scanning only longitudinal modes corresponding to Fabry-Pérot wavelengths were observed. The laser mode spectrum was measured at different driving currents for all vertical positions of the fiber tip. A corresponding current dependence taken in the vertical position $+300 \mathrm{~nm}$ is shown in Fig. 7. The longitudinal mode intensity of the peak at $919.8 \mathrm{~nm}$ increased with the driving current increase while the intensity of other modes lightly declines. In these current dependencies the additional peaks were observed for the current $I=55$ and $60 \mathrm{~mA}$. Their position in spectral dependencies does not correspond to the longitudinal Fabry-Pérot resonator wavelengths. Their behavior as well as origin was described in [9]. We suppose their relation with the higher-order lateral modes of a planar waveguide. Higher-order lateral modes are typically observable for current densities above kink region in the dependence of optical power vs.

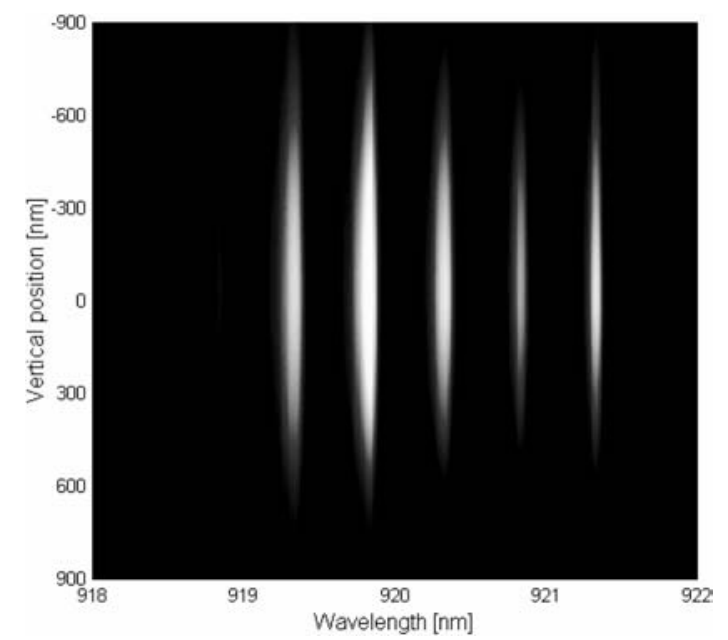

b)

Fig. 6 a) Laser mode spectrum at different vertical positions of the fiber tip at driving current $I=50 m A, b)$ interpolated density image.

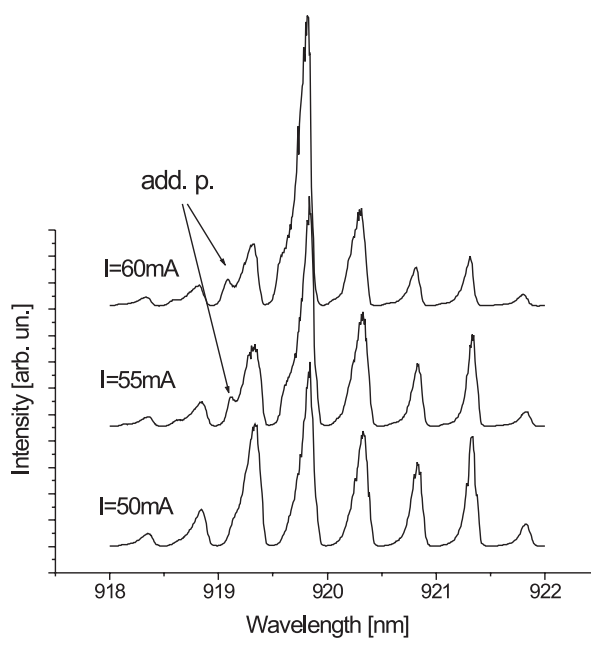

Fig. 7 The laser mode spectrum at different driving currents recorded at the vertical position $+300 \mathrm{~nm}$. First higher-order lateral modes are shown as weak additional peaks (add. p.).

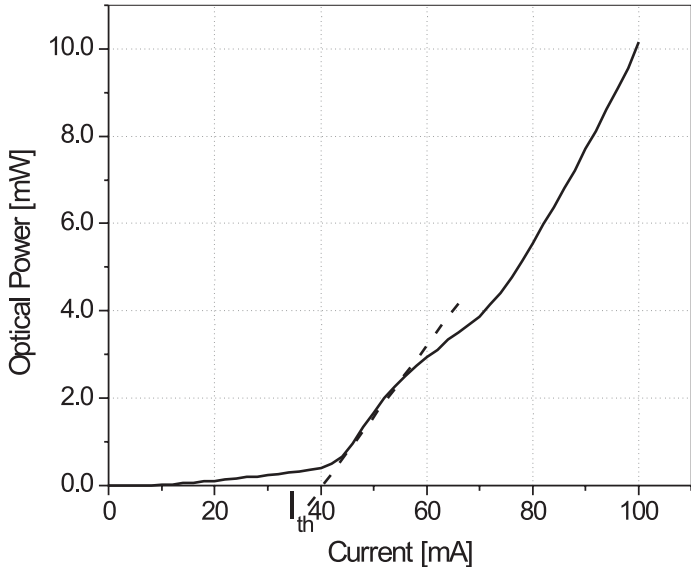

Fig. 8 Dependence of optical power vs. driving current with estimated threshold current value $I_{\text {th }}=40 \mathrm{~mA}$. 


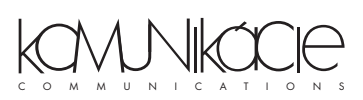

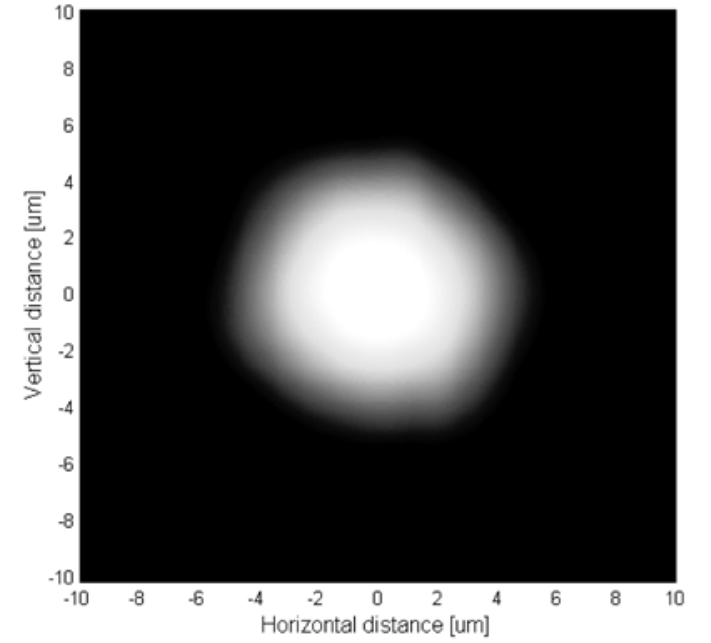

Fig. 9 The fundamental lateral mode in PCF reflects the hexagonal symmetry of the air holes spacing in the PCF structure. ture. However, the optical field of the $7 \mathrm{~cm}$ long PCF is a combination of the fundamental and the first higher-order lateral mode. All three possible symmetries of the first higher-order lateral mode are shown in Fig. 10.

\section{CONCLUSION}

The NSOM measurements using the prepared optical fiber tips could provide the high-resolution characterization of the optical field of semiconductor lasers. The NSOM was employed to obtain the near- and far-field profile of the InAs/GaAs MQW laser structures. From near- and far-field of the laser structure the parameters of optical field were determined. In the near-field region of InAs/GaAs MQW laser structures the spectrum was analyzed. From the locally analyzed spectra in the near-field the lateral modes in the spectrum were identified.

The NSOM experiment was employed for the near-field characterization of lateral modes in PCF. In the intensity image of the
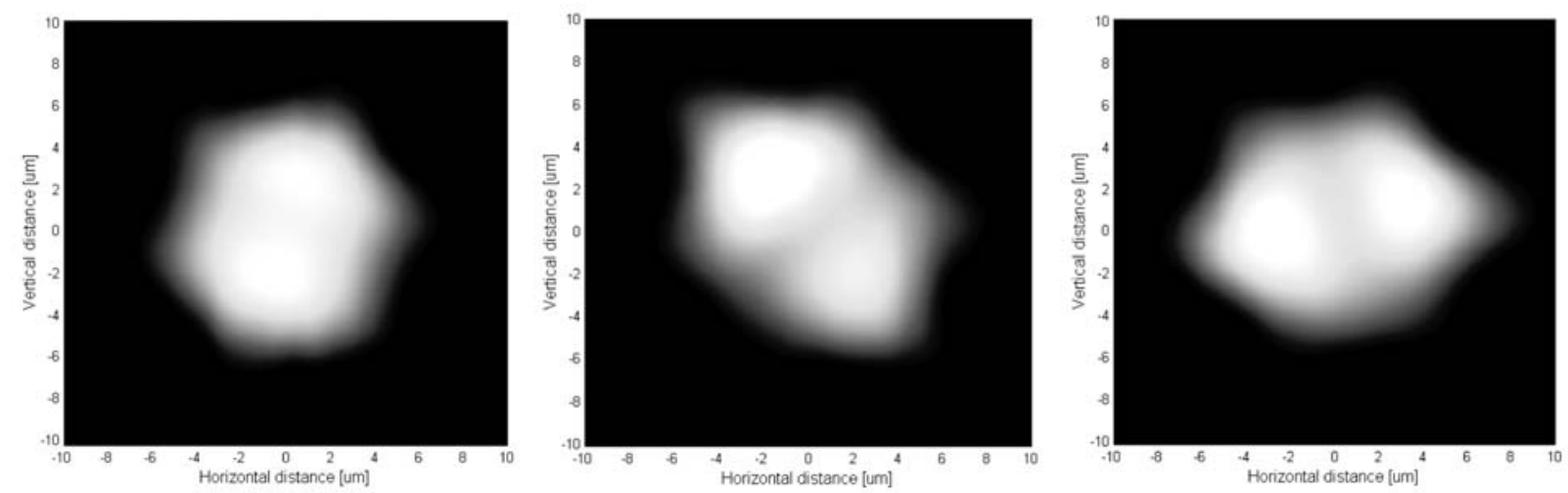

Fig. 10 Intensity image of the first higher-order lateral mode of PCF as a combination with fundamental lateral mode. All three possible symmetries of first higher-order mode are shown.

driving current [11]. It corresponds to the dependence of the optical power vs. driving current shown in Fig. 8.

The lateral modes in PCF were investigated by the horizontal and vertical scan. The intensity image of the scanned optical field in horizontal and vertical direction is shown in Fig. 9. According to the basic principles of wave propagation in optical fibers the radiation of higher-order lateral modes was expected for $150 \mathrm{~cm}$ long fiber [12]. Only fundamental lateral mode was observed for the $150 \mathrm{~cm}$ long fiber. The shape of the fundamental mode of the optical field reflects the hexagonal symmetry of the PCF struc-
PCF optical field the fundamental lateral mode as well as the first higher-order lateral mode was observed.

We expect that the NSOM technique will be a useful tool for waveguide optimization of semiconductor lasers and analysis of lateral modes in PCF.

\section{Acknowledgement}

This work was supported in part by Slovak National Grant Agency No. 1/2048/05, No. 1/0152/03, No. 1/0130/03 and by International Laser Centre.

\section{References}

[1] PAESLER, M. A., MOYER, P. J.: Near-field optics, (New York: John-Wiley\&sons, Inc.)., 1996, pp.21-31

[2] CAMPILlO, A. L., HSU, J. W. P., WHITE, C. A., JONES, C. D.: Appl. Phys. Letter, 80, 2002, p. 2239

[3] BRYANT, G. W., SHIRLEY, E. L., GOLDNER, ET AL., Phys. Rev. B, 58, 1998, p. 2131 
[4] HerZOG, W. D., ÜNLÜ, M. S., GOLDBERG, B. B., ET AL.: Appl. Phys. Letter 70, 1997, p. 668

[5] KOVÁČ, J., KVIETKOVÁ, J., KOVÁČ, J. Jr., ET AL.: Laser Physics, Vol. 14, No. 2, 2004, pp. 1-6

[6] LAEGSGARD, J., HANSEN, K. P., NIELSEN, M. D., ET AL.: In Proceedings of SBMO/IEEE MTT-S IMOC, 2003, pp. 259-261

[7] PUDIŠ, D., MARTINČEK, I., TUREK, I., ET AL.: In Proceedings of 10th International Workshop on Applied Physics of Condensed Matter, 2004, pp. 215-218

[8] PUDIS, D., MARTINCEK, I., TUREK, I., ET AL.: Will be published in Laser Physics, 15, No. 12, 2005

[9] PUDIŠ, D., MARTINČEK, I., KOVÁČ, J. JR., ET AL.: Advances in Electrical and Electronic Engineering, Vol. 4.,No. 2, 2005, pp. 97-100

[10] KÁČIK, D., TUREK, I., MARTINČEK, I. ET AL.: Intermodal interference in a photonic crystal fibre, Opt. Express 12, 3465-3470, 2004

[11] SINGH, J.: Optoelectronics, (NewYork: The McGraw-Hill Companies, Inc.), 1996

[12] GHATAK, A., THYAGARAJAN, K.: Introduction to fiber optics, Cambridge University Press, 1998, pp. 132-178.

Department of Fire Engineering, FŠI ŽU in Žilina

and

Secondary school of Fire Protection, MV SR in Žilina

Welcome you

$2^{\text {nd }}$ international conference

\section{FIRE PROTECTION AND RESCUE SERVICES}

\section{May 3. - 4. 2006 Žilina}

We are inviting you to the second meeting of professionals in the fire safety, which follows the conferences held in 2004

The conference will follow tradition and take place at the University of Žilina, Faculty of Special Engineering.

Contact address:

Ing. L’ubica Šovčíková - Conference grand

Department of Fire Engineering

Faculty of Special Engineering, University of Žilina

1. mája 32, 01026 Žilina

Slovak Republic

Tel.: 00421- 41-513 6799 Fax: 00421-41-513 6620

E-mail: lubica.sovcikova@fsi.utc.sk

Web site: www.fsi.utc.sk/kpi 